\title{
NEONATAL CANDIDIASIS IN ELBW AND VLBW INFANTS IN A UK TERTIARY NICU - 6 YEAR REVIEW
}

\author{
P. Siddhi ${ }^{1}$, S. Brown ${ }^{1}$, M. Patel ${ }^{2}$, M. Borooah $^{1}$ \\ ${ }^{1}$ Neonatal Intensive Care Unit (NICU) \\ ${ }^{2}$ Microbiology Department
}

\section{Introduction}

The overall incidence of invasive fungal infection is 2.4/1000 neonatal unit admissions. ${ }^{(1)}$ Extreme prematurity and Very Low Birth Weight (VLBW) babies are at a higher risk of invasive fungal infection. Apart from being immunecompromised, the invasive procedures and therapies make them more susceptible to fungal infections during the first few weeks of life.

\section{Aim}

We aim to identify the frequency of risk factors and the outcomes associated with invasive candidiasis.

\section{Method}

Retrospective review of neonates $<1500 \mathrm{~g}$ with positive blood culture for candida over 8 years (2009 - 2017). Data was obtained from case notes and electronic database. A universal policy of antifungal prophylaxis for VLBW in neonatal intensive care admissions was only introduced halfway through the study period.

Results

Median gestational age was 25 weeks (23-29 weeks). Median birth weight was $750 \mathrm{~g}(600-1460 \mathrm{~g})$. Median day of candidiasis was on day 17 of life. 9/12 (75\%) were identified to have Candida albicans. Other identified species included Candida parapsilosis.

Risk factors (Table-1)

$8 / 12$ (67\%) were on broad-spectrum antibiotics when identified to have candidiasis. 6/12 (50\%) had no antifungal prophylaxis. 9/12 (75\%) were colonised prior to positive blood culture. $10 / 12$ infants (83\%) had central lines. Mortality

6/12 (50\%) neonates with candidiasis died. Of them, five deaths were directly related to invasive candidiasis.

Table 1- Risk factors associated with Invasive candidiasis

\begin{tabular}{|ll|}
\hline Risk factors associated with Candidiasis & N (\%) \\
\hline Total number on broad spectrum antibiotics & $8(67 \%)$ \\
\hline No fungal prophylaxis & $6(50 \%)$ \\
\hline CVL & $10(83 \%)$ \\
\hline Skin colonization & $9(75 \%)$ \\
\hline Surgery & $5(42 \%)$ \\
\hline Invasive Ventilation & $6(50 \%)$ \\
\hline H2-Receptor Antagonist & $4(33 \%)$ \\
\hline Median antibiotic exposure days (Range 2-33 days) & 15 days \\
\hline
\end{tabular}

Fig 1. Timing of Invasive candidiasis (age in days)

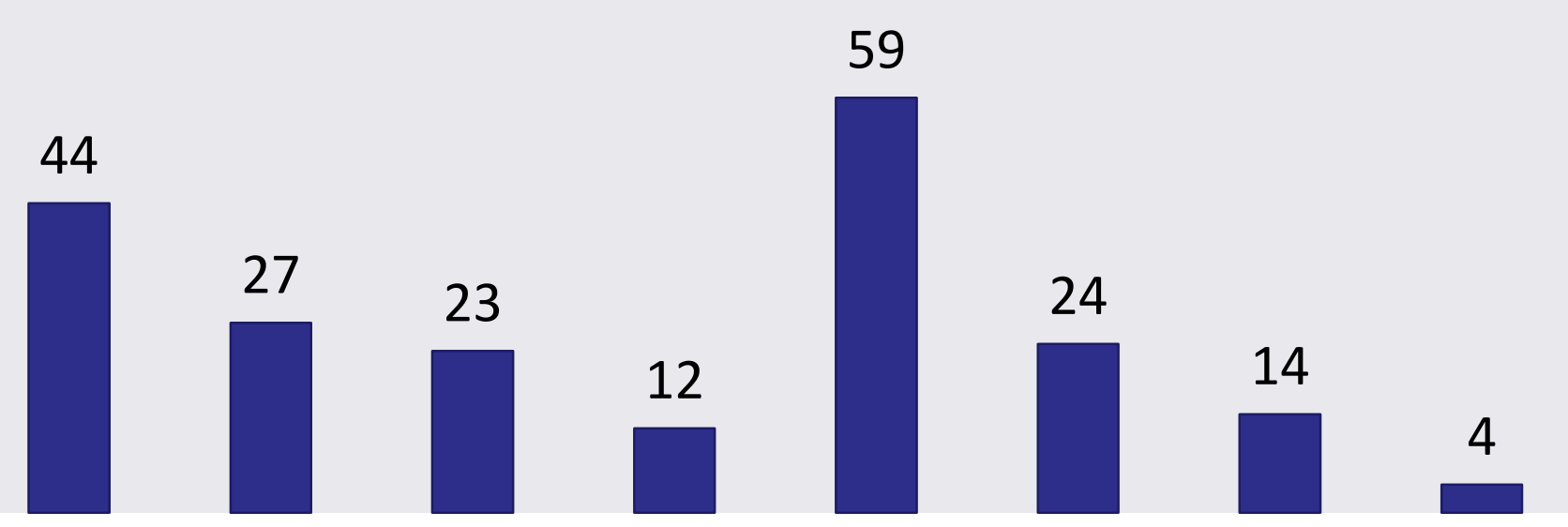

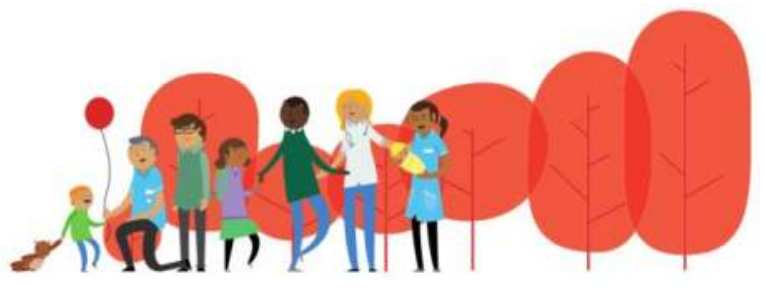

NTS

Birmingham Women's and Children's NHS Foundation Trust

One died later on but before discharge due to other co- morbidities. Of those neonates who died all 6 had platelet counts $<100 * 10^{9} /$.

\section{Conclusion}

Candida albicans was the commonest cause of invasive candidiasis. 11/12 $(92 \%)$ presented as late onset sepsis. The mortality rate is $50 \%$ in this cohort. The end organ involvement with neonatal candidaemia is low $(<20 \%)$ but the associated mortality is $100 \%$.

Table 2 - Results of the Imaging modalities used to identify end organ involvement

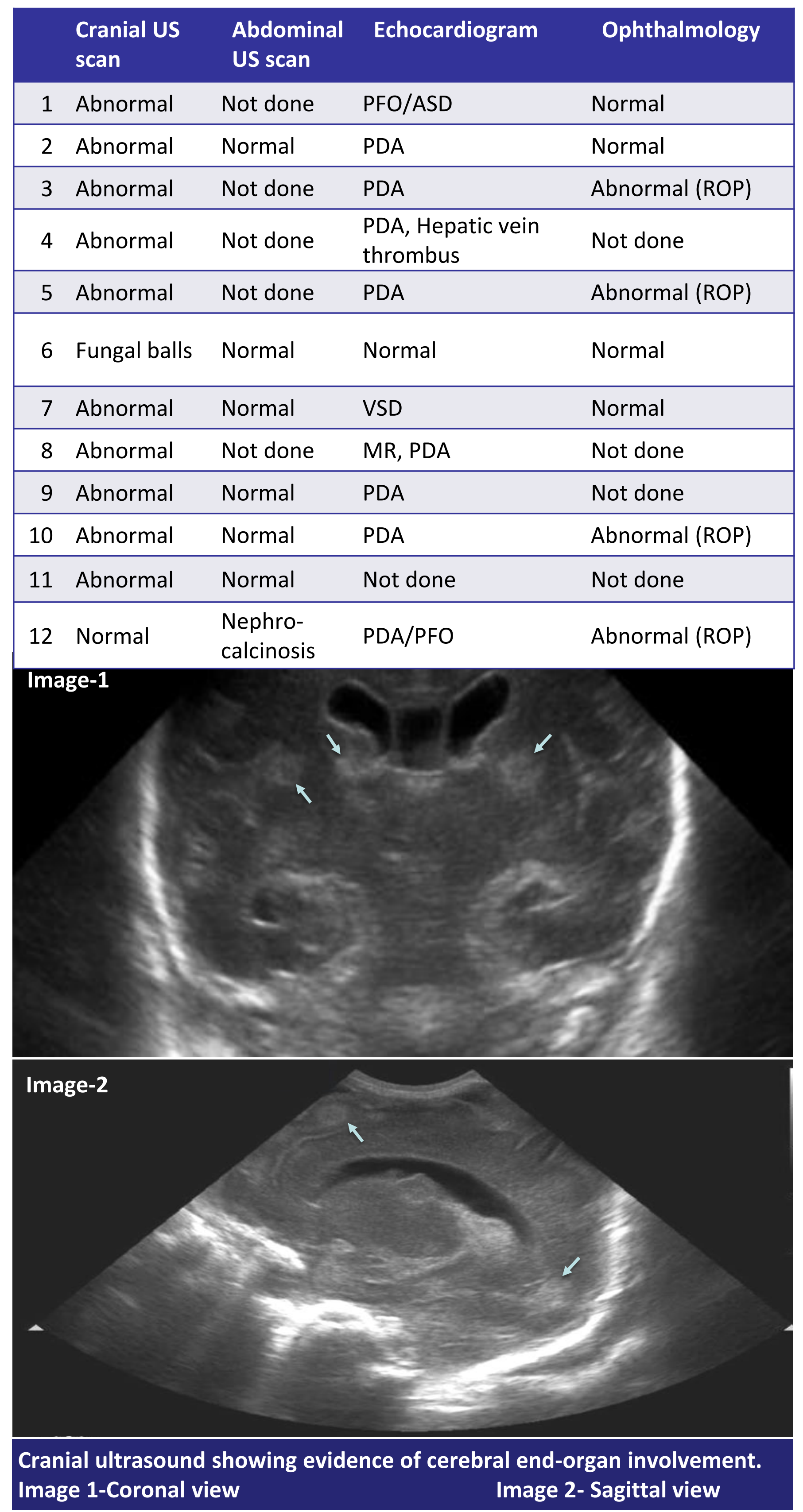

[Reference-1] C. Oeser, et al. Neonatal invasive fungal infection in England 2004-2010 Neonatal Infection Surveillance Network. Clinical Microbiology and Infection 2014 European Society of Clinical Microbiology and Infectious Diseases. 